03

\title{
О происхождении цепочек каверн во вращающемся потоке между цилиндрами
}

\author{
(C) Э.Л. Амромин \\ Federal Way WA 98003, СШA \\ e-mail: Amromin@aol.com
}

Поступило в Редакцию 25 апреля 2021 г.

В окончательной редакции 14 июня 2021 г.

Принято к публикации 4 июля 2021 г.

\begin{abstract}
Кавитация между вращающимся и неподвижным цилиндрами возникает в виде цепочки пузырьков. Размеры пузырьков практически одинаковы, равно как и расстояния между пузырьками и их азимутальное расположение. Хотя такая форма кавитации наблюдалась в многочисленных экспериментах (в частности, в экспериментах с подшипниками), ее природа не была выяснена. Проведенный анализ показывает, что нарушение осевой симметрии потока из-за смещения оси одного из цилиндров приводит к регулярным волнообразным трехмерным возмущениям потока. Их „длина волны“ определяется минимальным зазором между цилиндрами. Хотя течение между цилиндрами не является безвихревым, эти возмущения можно определить с помощью потенциала скорости.
\end{abstract}

Ключевые слова: кавитация, круговые цилиндры, несоосные цилиндры, невязкий поток.

DOI: 10.21883/JTF.2021.11.51523.119-21

\section{Введение}

Настоящая работа посвящена эксперименту [1], в котором обнаружена периодическая цепочка пузырьков, возникающих в потоке между несоосными цилиндрами. Эта периодичность не объяснена. О периодических пятнах зарождающейся кавитации также сообщалось в исследованиях [1-8] и обзорах $[9,10]$, касающихся течений в подшипниках. Такие пузырьки появляются немного ниже линии минимального зазора между цилиндрами. Аналитических исследований, объясняющих существование таких образований, в литературе не найдено.

Таким образом, здесь анализируется течение между двумя круговыми цилиндрами с параллельными осями. Схема потока на рис. 1 соответствует эксперименту [1]. Внешний цилиндр радиуса $R$ вращается с частотой $\Omega,(R$ будет использоваться ниже как единица длины, $\Omega R-$ как единица скорости). Радиус внутреннего неподвижно-

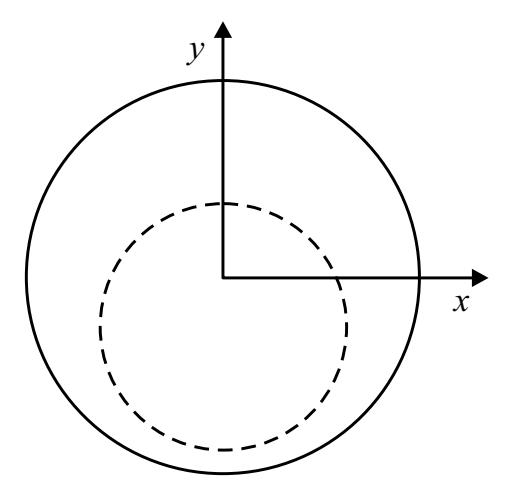

Рис. 1. Вид поперечного сечения потока $z=$ const; сплошным кружком показаны сечения вращающегося цилиндра, пунктиром - неподвижный цилиндр. го цилиндра равен $R_{i}$. Одна из осей цилиндра совпадает с осью $z$. Другая находится на расстоянии $\hat{y}$ ниже первой. Ось ординат проходит по минимальному зазору между цилиндрами. Экспериментальные данные [1] относятся к внешнему цилиндру радиуса $0.025 \mathrm{~m}$, вращающемуся с частотами в диапазоне $0.03<\Omega<0.34 \mathrm{~s}^{-1}$.

Традиционная математическая модель потоков между цилиндрами основана на рассмотрении уравнения Рейнольдса для гидродинамической смазки, но это уравнение зависит только от двух переменных. Между тем, возникновение зазора между осями цилиндров приводит к трансформации осесимметричного потока в трехмерный, и становится необходимым учитывать трехмерные возмущения потока. Хотя невозмущенный поток не является безвихревым, его возмущения можно определить с помощью потенциалов скорости, как это было описано в $[11,12]$ для других потоков с видимой регулярной структурой.

\section{1. Расчет невязкого потока}

Рассмотрим течение между несоосными цилиндрами как возмущенное установившееся течение между соосными цилиндрами. Будут использоваться две цилиндрические системы координат. Система $\{z, r, \theta\}$ связана с внешним цилиндром, система $\left\{z, r^{*}, \varphi\right\}-$ с внутренним цилиндром; $\theta$ и $\varphi$ отсчитываются от линии симметрии сечения. Невозмущенное течение можно описать приближенной формулой

$$
u_{0}\{r, \theta\}=\left(r-\frac{\varepsilon^{2}}{r}\right) \frac{1-R_{i}^{2}\left(1-2 \ln R_{i}\right)}{1-\varepsilon^{2}+2 \varepsilon^{2} \ln \varepsilon}
$$

для окружной скорости в потоке между цилиндрами, где $\varepsilon(\theta)=1-h(\theta), h-$ расстояние (зазор) между 
поверхностями цилиндров. Эта формула удовлетворяет закону сохранения массы. Поток жидкости через сечения $\theta=$ const между коаксиальными цилиндрами равен $0.5\left[1-R_{i}^{2}\left(1-2 \ln R_{i}\right)\right] /\left(1-R_{i}^{2}\right)$. В уравнении (1) предполагается, что тот же поток будет сохраняться после смещения оси внешнего цилиндра, а также будет сохраняться подобие профиля скорости вдоль продолжения радиуса внутреннего цилиндра. Значение $h$ отсчитывается вдоль этого продолжения. Также можно найти из уравнения (1) скорость невозмущенного потока на поверхности внутреннего цилиндра, равную нулю. На внешнем она растет с уменьшением $h$.

Трехмерное возмущение скорости можно определить с помощью потенциала скорости $\Phi$, состоящего из двух компонент, которые являются решениями уравнения Лапласа (к которому сводится тот же закон для несжимаемых жидкостей). Первая компонента

$$
\Phi_{1}=A \sin (\theta) \cos (k z) I_{1}(k r),
$$

где $I_{1}$ - модифицированная функция Бесселя, является фундаментальным решением [13] уравнения Лапласа; волновое число $k$ находится из условия

$$
\frac{d I_{1}}{d r}(k)=0 \text {. }
$$

У уравнения (3) много корней. Выбор между ними должен производиться с учетом дополнительного условия $\frac{d I_{1}}{d r}(k-k H) \simeq 0$. Здесь $H-$ минимальный зазор между цилиндрами. Вторая компонента

$$
\Phi_{2}=\frac{1}{4 \pi} \oiiint_{S_{0}+S_{i}}^{\square} \frac{Q d s}{D}
$$

определяется с помощью источников или стоков интенсивности $Q$, распределенных по поверхностям обоих цилиндров. Здесь $S_{0}$ и $S_{i}-$ поверхности внешнего и внутреннего цилиндров, расстояние $D=\sqrt{(z-\zeta)^{2}+\left(\sin \theta-R_{i} \sin \varepsilon\right)^{2}+\left(\cos \theta-R_{i} \cos \varphi-\hat{y} \cos \varphi\right)^{2}}$. Граничными условиями для определения интенсивности $Q$ являются условия непротекания обеих поверхностей. Это условие имеет вид

$$
\frac{\partial \Phi_{2}}{\partial r}+\frac{\partial \Phi_{1}}{\partial r}=0
$$

на $S_{0}$, тогда как на $S_{i}$

$$
\frac{\partial \Phi_{2}}{\partial r^{*}}+\frac{\partial \Phi_{1}}{\partial r^{*}}+u_{0} \cos (\widehat{r, \theta})=0 .
$$

Дополнительное условие

$$
\oiiint_{S_{0}}^{\square}\left[\frac{\partial \Phi_{1}}{\partial r}+\frac{1}{4 \pi} \frac{\partial}{\partial r} \oiiint_{S_{1}}^{\square} \frac{Q d s}{D}\right] d s=0
$$

позволяет определить коэффициент $A$. Решение этой задачи для уравнения Лапласа может быть найдено методом граничных элементов. Наличие точных формул для

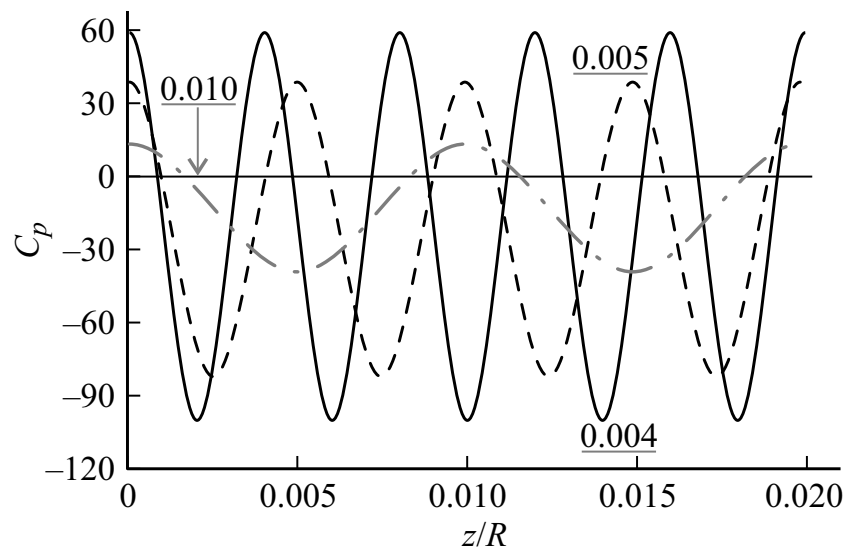

Рис. 2. Распределение давления по линии минимального зазора между цилиндрами для $R_{i}=0.75 R$; числа у кривых показывают значения $H$; коэффициент давления нормирован на $0.5 \rho(\Omega R)^{2}$.

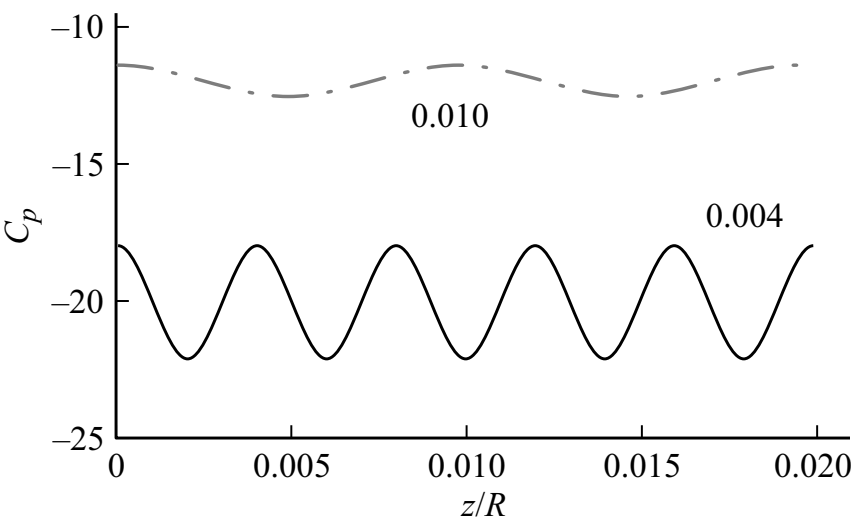

Рис. 3. Распределение давления по линии минимального зазора между цилиндрами для $R_{i}=0.95 R$; числа у кривых показывают значения $H$; коэффициент давления нормирован на $0.5 \rho(\Omega R)^{2}$.

координат $S_{0}$ и $S_{i}$ позволяет избежать аппроксимации этих поверхностей (в отличие от алгоритма, описанного, например, в [14]) и тем снизить ошибки вычислений. После вычисления компонент скорости давление может быть вычислено с помощью интегрирования уравнения Навье Стокса, записанного в цилиндрических координатах (см. Приложение 2 в [15]). Однако в минимальном зазоре между цилиндрами радиальная составляющая скорости пренебрежимо мала, а ее азимутальная составляющая имеет максимум. Поэтому при интегрировании можно опустить члены, зависящие от вязкости.

Сравнения расчетного распределения давления по поперечным сечениям внешних линдров $(z=$ const $)$ для двух значений $R_{i}$ и нескольких $H=1.0-R_{i}-\hat{y}$ приведены на рис. 2 и 3 для разницы между $R$ и $R_{i}$, много меньшей, чем в эксперименте [1], но это ближе к ситуации с подшипниками [9]. Как видно из этих рисунков, вклад $\Phi_{1}$ приводит к осевым и радиальным волнам давления, упомянутым в [2]. Как можно заключить из 


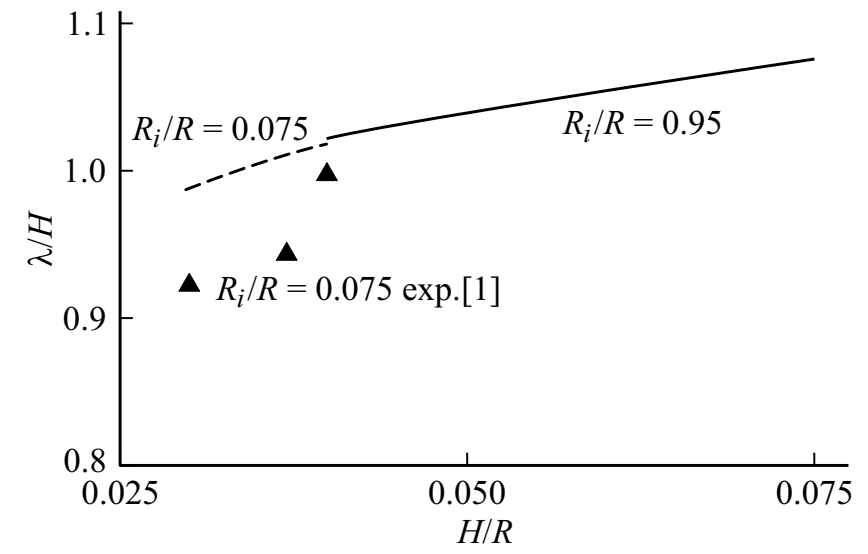

Рис. 4. Зависимости длин волн от минимальных зазоров между цилиндрами. Линии - расчеты, треугольники - экспериментальные данные [1].

приведенных выше расчетов, постоянное расстояние между двумя параллельными осями цилиндров приводит к генерации „волн“. В окрестностях минимумов давления должны находиться возникающие каверны.

Сравнение рассчитанных зависимостей длины волны $\lambda$ с измеренными в [1] представлено на рис. 4. Их различие можно оценить как удовлетворительное. Расчетная кривая для другого отношения $R_{i} / R$ и соответственно других $\hat{y}$ на том же графике (штриховая кривая) практически совпадает с первой. Итак, разработанная математическая постановка задачи позволяет объяснить природу наблюдаемых систем полостей.

\section{2. О прогнозе возникновения кавитации}

Исследуемые регулярные пятна кавитации появлялись в очень широком диапазоне чисел Рейнольдса, в том числе при достаточно высоких $\operatorname{Re}\left(\right.$ при $\operatorname{Re}>10^{6}$ в [6]). В частности же в экспериментах [1], проведенных с жидкостью PDMS1000, имеющей кинематическую вязкость $v=10^{-3} \mathrm{~m}^{2} \mathrm{~s}^{-1}$, соответствующие значения Re ниже 1.0 . Для анализа этих экспериментов модели кавитационных течений вязкой жидкости, использованные в [16] (но ранее предложенные в [17]) или в [18] (но ранее предложенные в [19]), не подходят.

Таким образом, автор сравнивает свои результаты с экспериментальными данными [1] по возникновению кавитации, используя очень приблизительные оценки. Во-первых, им было принято, что полости возникают в виде сферических пузырьков вблизи точек отрыва ламинарного пограничного слоя от цилиндра. Во-вторых, было принято, что давление в жидкости в этих местах можно оценить без учета обратного влияния на нее зоны отрыва (как в устаревшем методе, использованном в [20]). В-третьих, поскольку известные критерии отрыва ламинарного пограничного слоя не работают при столь малых $\operatorname{Re}($ как и специальный критерий из [10] для потоков между цилиндрами), точка максимального градиента давления была выбрана в качестве точки отрыва пограничного слоя. Тем не менее вычисления хорошо согласуются с экспериментальными данными [1]: точка максимума градиента находится на 6.5 градусов ниже минимума зазора, тогда как наблюдаемые пузырьки располагались примерно под 7 градусами. Пример расчетного распределения давления по сечению внутреннего цилиндра в идеальной жидкости показан на рис. 5. Число кавитации в [1] не вводилось и сравнение чисел возникновения кавитации невозможно.

Итак, удовлетворительное решение для рассматриваемого вида отрывных течений было найдено в рамках теории идеальной жидкости (как и в ситуациях, рассмотренных в [21]). Обычно расчеты внутренних кавитирующих потоков для очень малых чисел Рейнольдса выполняются с использованием программного обеспечения для уравнений Навье-Стокса (как в [22]). Имеется единственная работа [23], посвященная кавитации между цилиндрами при таком Re, однако там рассматривалась достаточно развитая кавитация, и должно быть сглаживающее обратное влияние каверны на периодические минимумы давления. Таким образом, нет возможности сравнения результатов этой работы с результатами [23].

Точный количественный анализ при значениях Re, соответствующих данным [1], может быть возможен с помощью метода прямого численного моделирования (Direct Numerical Simulation, DNS). Однако в анализ необходимо включить силу поверхностного натяжения, поскольку, согласно фото [1], для $H=0.004$ радиус появляющихся пузырьков приблизительно равен $R_{C}=0.00004 \mathrm{~m}$. Поскольку коэффициент поверхностного натяжения, нормализованный с использованием плотности PDMS1000, составляет $\chi=0.02 \mathrm{~m}^{3} \mathrm{~s}^{-2}$ при $20^{\circ} \mathrm{C}$, отношение силы инерции в месте расположения пузыря к силе поверхностного натяжения составляет приблизительно 1.26. Следовательно, здесь существенно влияние силы поверхностного натяжения. В результате существующие DNS методы для кавитирующих потоков без

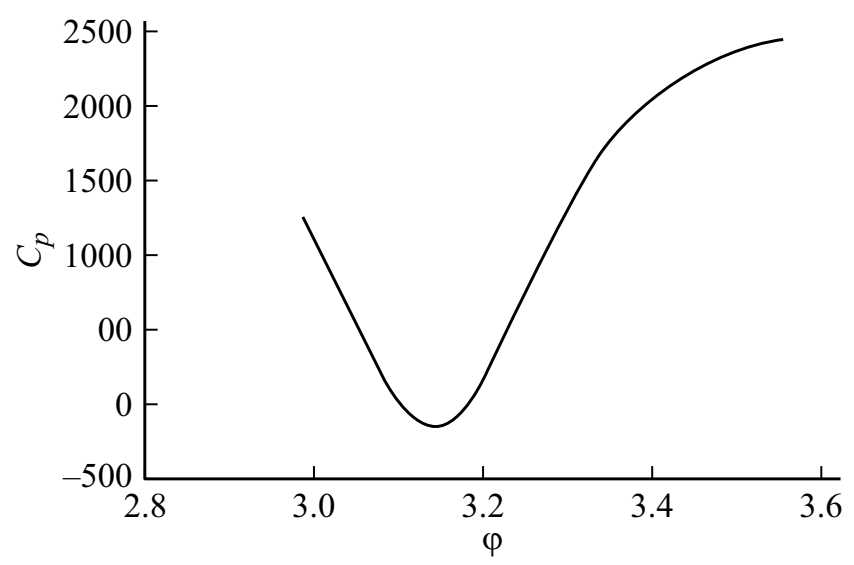

Рис. 5. Пример расчетного распределения давления по поперечному сечению внутреннего цилиндра $(H=0.005)$; коэффициент давления нормирован на $0.5 \rho(\Omega R)^{2}$. 
учета поверхностного натяжения (как описано в [24]) должны быть модифицированы, чтобы учесть эффект поверхностного натяжения.

\section{Заключение}

Периодическая цепочка пузырьков в потоках между цилиндрами описывается с использованием потенциала скорости для неосесимметричных возмущений этих потоков. Показано, что постоянное расстояние между двумя параллельными осями цилиндров приводит к возникновению волнообразных распределений давления вдоль них. Регулярная система минимумов давления приводит к появлению регулярной системы каверн с практически постоянными расстояниями между ними. „Длина волны“ системы определяется минимальным зазором между цилиндрами. Сравнение расчетов с экспериментальными данными показало способность разработанной расчетной модели удовлетворительно прогнозировать азимутальное и осевое расположение возникающих каверн. Итак, можно заявить, что природа изучаемой цепочки обнаружена. Хотя описанная модель потока не позволяет количественно прогнозировать число возникновения кавитации, проведенные расчеты позволяют сделать вывод, что отклонение от осесимметричного потока (расстояние между осью внутреннего цилиндра и осью внешнего) является более существенным для возникновения кавитации, чем увеличение скорости вращения, и, поскольку эти отклонения по существу трехмерны, их влияние нельзя проанализировать с помощью уравнения Рейнольдса для смазки.

Наконец, необходимо отметить, что модели течений жидкости, разработанные профессором Степановым (в 2022 г. ему исполнилось бы 100 лет), остаются эффективными для решения задач теории кавитации.

\section{Конфликт интересов}

Автор заявляет, что у него нет конфликта интересов.

\section{Список литературы}

[1] A.A. Monakhov, V.M. Chernyavski, Y. Shtemler. Phys. Fluids, 25, 093102 (2013).

[2] A. Harnoy. Bearing Design in Machinery (Taylor \& Francis. Bosa Roca, US, 2002)

[3] V.R. Nosov, J. Gomez-Mancilla, J.A. Meda-Campana. Water Sci. Techn., 64, 593 (2011).

[4] T. Tang, N. Morris, J. Coupland. J. Physics: Conference Series, 656, 012119 (2015).

[5] V. Shinde, N. Padawale, H. Tambat. ARPN J. Eng. Appl. Sc., 11, 7936 (2016).

[6] Y. Song, X. Ren. ASME J. Tribology, 137, 011701 (2015).

[7] D.Y. Dhande, D.W. Pande. J. King Saud University - Eng. Sc., 30, 345 (2018).

[8] P. Reinke, M. Schmidt, T. Beckmann. Phys. Fluids, 30, 104101 (2018).
[9] D. Dowson, C.M. Taylor. Ann. Rev. Fluid Mech., 11, 35 (1979).

[10] M.J. Braun, W.M. Hannon. Proceedings of the Inst. Mech. Eng., Part J: J. Eng. Tribology, 224, 839 (2010).

[11] E.L. Amromin, S.I. Kovinskaya. J. Fluids Structures, 34, 84 (2012).

[12] E.L. Amromin. Intern. J. Multiphase Flows, 124, 103188 (2020).

[13] Г. Корн, Т. Корн. Справочник по математике (Наука, М., 1970)

[14] Э.Л. Амромин, Г.Ю. Степанов, Ю.С. Тимошин. ЖТФ, $65(10), 13$ (1995).

[15] Д. Бэтчелор. Введение в динамику жидкости (Мир, М., 1973)

[16] Э.Л. Амромин. МЖГ Известия АН СССР, 20, 871 (1985).

[17] Г.Ю. Степанов. Избранные вопросы современной механики (ИМ МГУ, 1982), с. 5.

[18] O. Coutier-Delgosha, F. Deniset, J.A. Astolfi, J.-B. Leroux. ASME J. Fluids Eng., 129, 279 (2007).

[19] Л.В. Гогиш, Г.Ю. Степанов. Отрывные и кавитационные течения (Наука, М., 1990)

[20] M.L. Billet, J.W. Holl. ASME J. Fluids Eng., 103, 405 (1981).

[21] Г.Ю. Степанов. Труды МИАН, 186, 31 (1989).

[22] S. Dabiri, W.A. Sirignano, D.D. Joseph. Physics of Fluids, 22 (4), 042102 (2010).

[23] А.А. Монахов, В.Д. Котелкин. Изв. РАН. МЖГ, 3, 81 (2017).

[24] A. Znidarcic, O. Coutier-Delgosha, M. Marquillie, M. Dular. J. Physics: Conf. Series, 656, 012143 (2017). 